\title{
Ocean surface current retrieval from space: The Sentinel-2 multispectral capabilities
}

\author{
Yurovskaya Maria ${ }^{1,2,{ }^{*}}$, Kudryavtsev Vladimir ${ }^{1,2}$, Chapron Bertrand ${ }^{2,3}$, Collard Fabrice ${ }^{4}$
}

\author{
${ }^{1}$ Marine Hydrophysical Institute of RAS, Sevastopol, Russia \\ 2 Satellite Oceanography Laboratory, Russian State Hydrometeorological University, Saint Petersburg, \\ Russia \\ 3 Institut Français de Recherche pour l'Exploitation de la Mer, Plouzané, France \\ ${ }^{4}$ OceanDataLab, Déolen, France
}

Maria Yurovskaya, email address : $\underline{\text { mvkosnik@gmail.com }}$

\begin{abstract}
:
The Sentinel-2 MultiSpectral Instrument (MSI) collects multiple spectral band images, corresponding to specific sensing wavelengths and spatial resolutions, i.e. $10 \mathrm{~m}, 20 \mathrm{~m}$ and $60 \mathrm{~m}$, respectively. Images are collected one at the time with a given time-lag between observations. Under favorable conditions, spatiotemporal characteristics of propagating ocean surface waves can thus uniquely be retrieved. A method for surface current vector field reconstruction is then developed. Demonstrated over different deep ocean regions, the retrieved surface current fields well compare with medium-resolution ocean circulation model or derived-velocities from altimeter measurements. At finer scales, the surface wave-conservation law is recovered, with the associated relationship between current vorticity and wave-ray curvature. Over shallow water regions, the wave propagation properties well follow sea depth variations, consistent with ETOPO1 data. Finally, time-lag between detector bands can also be exploited to estimate speed and direction properties of detected surface wave breaking whitecaps. An analysis of velocity reconstruction errors further reveals that Sentinel-2 MSI inter-channel co-registration is realized with an accuracy better than 0.1 pixel. Overall, these results confirm very promising capabilities of optical imagery to provide direct surface current velocity measurements from space, over relatively large areas, $\mathrm{O}(100 \mathrm{~km})$, with a spatial resolution down to $\mathrm{O}(1 \mathrm{~km})$.
\end{abstract}

\section{Highlights}

- A method is developed for surface current reconstruction from Sentinel-2 images $>$ It is based on cross-spectral phase estimation between images from different bands Retrieved current fields well compare with model and altimeter measurements Over shallow water regions, sea depth is reconstructed Inter-channel time delay is used to estimate speed and direction of large whitecaps

Keywords : Ocean currents, Sea surface optical images, Wave dispersion relation, Time lag, Wave breaking, Sentinel-2, Satellite methods 
An area of unfilled promise in satellite ocean remote sensing is the development of a direct inversion of upper ocean surface current characteristics.

From precise sea level measurements, satellite altimeters greatly help reveal balanced motions to describe large to meso-scale ocean dynamics properties. Indeed, away from the equator, a sea level gradient results as a pressure gradient, to be balanced by the Coriolis force associated to a current flow. This is the geostrophic balance. Altimeter-derived currents thus provide a good representation of large scale depth-integrated currents. However, surface currents are certainly not always in geostrophic balance, and also reflect interactions with upper ocean wind and wave motions. Altimeter measurements cannot fully capture multi-scale variability of the ocean circulation, especially within the uppermost surface layers, which remains a challenge for theoretical and practical ocean modeling. Techniques using Doppler radar measurements (e.g. Goldstein and Zebker (1987); Chapron et al. (2005); Romeiser et al. (2014); Rodriguez et al. (2018)) can provide more direct measurements, but also face a number of difficulties. Not solely related to 
platform attitude issues, inversion algorithm definitions must account for detected orbital motions of waves, including wave breaking effects (Johannessen et al. (2008); Hansen et al. (2012); Martin and Gommenginger (2017); Martin et al. (2018); Yurovsky et al. (2019)).

Overlooked, techniques from photographs and video recordings of the sea surface have long been demonstrated and reported in various field studies (e.g., Barber (1949); Dugan et al. (2001); Dugan and Piotrowski (2003); Leckler et al. (2015); Rascle et al. (2017); Yurovskaya et al. (2018)). Today, with the significant improvement of optical instruments, like event cameras (Rebecq et al. (2017)), stereo systems (Fedele et al. (2013)) and relatively simple remote controlled measurements from drones (Yurovskaya et al. (2018a); Yurovskaya et al. (2018)), improved spatio-temporal properties of the ocean surface can be readily obtained.

From a satellite perspective, optical imagery also provides high resolution sea surface images over large areas. As presently available, an ensemble of satellite missions (e.g Landsat-8, Sentinel-2a and 2b, RapidEye, among others) not only often enable to distinguish individual ocean surface waves from acquired images, but also provide means to characterize spatio-temporal ocean surface wave displacements. Indeed, the specific viewing geometry of the satellite radiometers offers time-lagged acquisitions for different spectral channels. Near-simultaneous measurements can thus be exploited to estimate local displacements, such as moving objects on the sea surface, river ice debris (Kääb et al. (2013)), ships (Takasaki et al. (1994)), surface wave fronts (De Michele et al. (2012)), but also wave breakers that often manifest in satellite optical images as localized bright events. An overview of motion 
detection methods from near-simultaneous satellite images is given in Kääb and Leprince (2014).

For Sentinel-2 measurements, cross-spectral analysis between images collected in two specific bands, was first demonstrated to retrieve surface wave directional properties and current characteristics (Kudryavtsev et al. (2017a,b)). This initial methodology was then further improved to reconstruct the surface current velocity vector (Yurovskaya et al. (2018b)). In this present work, the aim is to further test the instrument capabilities, applying the proposed methodology for different cases and over different ocean regions. For deep sea cases, the analysis concentrates on the applicability of the principle of wave action conservation, and more specifically the expected relationship between current vorticity and wave-ray curvature (Kenyon (1971); Quilfen et al. (2018)). Over shallow water regions, changes of wave dispersion properties are then further shown to help reveal sea depth variations. Finally, time-lag between detector bands is further exploited to estimate, from space, speed and direction of detected surface wave breaking whitecaps, key for direct measurements of momentum and gas fluxes.

The structure of the paper is as follows. Section 2 introduces Sentinel2 MSI time-lagged measurements, and the method to retrieve current and bathymetry information. Examples, including whitecap velocities, are given in Section 3. Discussion in Section 4 covers applicability of the proposed approach, possible error sources and estimation of velocity reconstruction accuracy. 


\section{Sentinel-2 MSI data and Methodology}

\subsection{MSI time-lagged data}

Sentinel-2A and Sentinel-2B multispectral (MSI) instruments are composed of 13 spectral bands (443-2190 nm) with different spatial resolutions, ranging from $10 \mathrm{~m}$ to $60 \mathrm{~m}$, to cover a swath width of $290 \mathrm{~km}$ formed by 12 sensor clusters. Due to a specific sensor configuration, i.e. the relative detector alignment, there is a parallax angle between both neighbor clusters and spectral channel sensors inside each cluster. It leads to inter-cluster and inter-band short-time lags that can be estimated from a viewing geometry as

$$
\begin{array}{r}
d t=D / V \\
D=H \sqrt{\tan ^{2} \theta_{1}+\tan ^{2} \theta_{2}-2 \tan \theta_{1} \tan \theta_{2} \cos \left(\varphi_{1}-\varphi_{2}\right)},
\end{array}
$$

where $H$ is the altitude of the satellite, $\theta_{1,2}$ and $\varphi_{1,2}$ zenith and azimuth view angles of the same point in two different bands, $V$ is the satellite speed and $D$ the length of satellite track. Compared to Eq. (22) by Kudryavtsev et al. (2017a), the absolute satellite speed and flight distance are considered in Eq. (1), instead of ground ones, as the viewing angles are defined from ground to sensor.

While this is not critical for an overall estimation of wave dispersion characteristics (Kudryavtsev et al. (2017a,b)), a more precise $d t$ value is essential to best retrieve local velocities, as discussed in Section 4.

Values for $H$ and $V$ are calculated from satellite coordinates, given in MSI auxiliary files. The time values for each band and strip (cluster) are also directly listed in metadata information. As tested, both ways to estimate the time lags give similar results, within 1-2\% precision. The summary of 
inter-band and inter-cluster relative time shifts is reported in Fig. 1. The listed time lags apply for both Sentinel-2A and Sentinel-2B data at different satellite locations.

Time-lagged acquisitions can be employed to infer instant velocity estimates. For long enough time delay, a surface drift can be estimated by tracking detectable features, e.g. surface slicks, as performed by Matthews and Awaji (2010) and Matthews and Yoshikawa (2012), using 45 s timelagged satellite stereo acquisitions. The larger the time lag, the more accurate becomes the motion detection (Kääb and Leprince (2014)). For ocean surface wave dispersion analysis, the time lag should be shorter, to ensure high coherency between two consecutive observations. Optimal values can still amount to several seconds. As estimated for MSI, the longest inter-band time lag is 2.6 s, obtained for B02 and B09 data. The B09 spatial resolution $(60 \mathrm{~m})$ is too coarse to sufficiently resolve surface waves. For our purpose, preference is given to a higher resolution channel combination, i.e. bands B02 and B04 with 1 s time lag.

\subsection{Current Vector and Ocean Depth Reconstruction}

To derive wave dispersion characteristics, the approach follows Kudryavtsev et al. (2017a). Consider $I_{1}, I_{2}$, image brightness fragments in B02 and B04, respectively, their coherence and phase spectra are:

$$
\begin{gathered}
\text { Coh }=\frac{\operatorname{abs}\left(\hat{I_{1}} \hat{I}_{2}^{*}\right)}{\sqrt{\hat{I}_{1} \hat{I}_{1}^{*} \cdot \hat{I}_{2} \hat{I}_{2}^{*}}}, \\
F=\arg \left(\hat{I}_{1} \hat{I}_{2}^{*}\right),
\end{gathered}
$$


Inter-Detector Time Lag

\begin{tabular}{|c|c|c|c|c|c|c|c|c|c|c|c|}
\hline $\mathrm{B} 01(60 \mathrm{~m})-7^{\prime}$ & 0.3 & 7 & 0.3 & $7^{\prime}$ & 0.3 & $7^{\prime}$ & 0.3 & $7^{\prime}$ & 0.3 & $7^{\prime}$ & 0.3 \\
\hline $\mathrm{B} 02(10 \mathrm{~m})-4.7$ & 2.6 & 4.7 & 2.6 & 4.7 & 2.6 & 4.7 & 2.6 & 4.7 & 2.6 & 4.7 & 2.6 \\
\hline $\mathrm{BO}(10 \mathrm{~m})-5.2$ & 2.1 & 5.2 & 2.1 & 5.2 & 2.1 & 5.2 & 2.1 & 5.2 & 2.1 & 5.2 & 2.1 \\
\hline BO4 $(10 \mathrm{~m})-5.7$ & 1.6 & 5.7 & 1.6 & 5.7 & 1.6 & 5.7 & 1.6 & 5.7 & 1.6 & 5.7 & 1.6 \\
\hline B05 $(20 m)-6$ & 1.3 & 5.9 & 1.4 & 5.9 & 1.4 & 5.9 & 1.4 & 5.9 & 1.4 & 6 & 1.3 \\
\hline B06 $(20 \mathrm{~m})-6.2$ & 1.1 & 6.2 & 1.1 & 6.2 & 1.1 & 6.2 & 1.1 & 6.2 & 1.1 & 6.2 & 1.1 \\
\hline B07 $(20 m)-6.5$ & 0.8 & 6.5 & 0.8 & 6.4 & 0.9 & 6.4 & 0.9 & 6.4 & 0.8 & 6.5 & 0.8 \\
\hline BO8 $(10 m)-4.9$ & 2.4 & 4.9 & 2.4 & 4.9 & 2.4 & 4.9 & 2.4 & 4.9 & 2.4 & 4.9 & 2.4 \\
\hline B8A $(20 m)-6.8$ & 0.5 & 6.7 & 0.6 & 6.7 & 0.6 & 6.7 & 0.6 & 6.7 & 0.6 & 6.7 & 0.5 \\
\hline B09 $(60 m)-7.3$ & 0 & 7.3 & 0 & 7.2 & 0.1 & 7.2 & 0.1 & 7.2 & 0 & 7.3 & 0 \\
\hline B10 $(60 m)-5.6$ & 1.8 & 5.5 & 1.8 & 5.5 & 1.8 & 5.5 & 1.8 & 5.5 & 1.8 & 5.6 & 1.8 \\
\hline B11 (20m) -6.2 & 1.1 & 6.2 & 1.2 & 6.1 & 1.2 & 6.1 & 1.2 & 6.2 & 1.2 & 6.2 & 1.1 \\
\hline B12 $(20 m)-6.8$ & 0.5 & 6.8 & 0.5 & 6,8 & 0,5 & 6,8 & 0.5 & 6,8 & 0,5 & 6.8 & 0,5 \\
\hline 1 & 2 & 3 & 4 & ${ }^{5}$ & 6 & 7 & 8 & 9 & 10 & 11 & 12 \\
\hline
\end{tabular}

Figure 1: Inter-cluster and inter-band time lag (relative to the earliest acquisition) in MSI Sentinel-2 data.

with $\hat{I}_{1,2}$, Fourier transform, and ${ }^{*}$ its complex conjugation. Following linear surface wave theory, the phase spectrum in absence of current is

$$
F_{0}=d t \sqrt{g k \tanh (k h)},
$$

where $g$ is gravity, $k$ wavenumber, and $h$ water depth.

In presence of a surface current, the surface wave dispersion is modified. The upper ocean is moving with a current velocity $\mathbf{U}$, very slowly varying with position, i.e. varying over a scale much larger than the peak wavelength of the wave system, typically from $1 \mathrm{~km}$ to $20 \mathrm{~km}$. To first order, this surface current locally, e.g. $\mathrm{O}(1 \mathrm{~km})$, Doppler-shifts the phase as:

$$
F=F_{0}+\mathbf{k} \mathbf{U} \cdot d t
$$


In terms of phase velocity, $C=F /(k d t)$, a transect of the phase spectrum along the wave direction provides an estimate of the corresponding surface current projection, as a measurable deviation between $C\left(k_{j}\right)$ and $C_{0}\left(k_{j}\right)$ :

$$
U_{j}=C-C_{0}=F /(k d t)-\sqrt{\tanh (k h) g / k}
$$

or for deep sea conditions $(k h>>1)$ :

$$
U_{j}=F /(k d t)-\sqrt{g / k}
$$

For deep water conditions or when the ocean depth is known, the inclination of the plane $\left(F-F_{0}\right) / d t$ in the wavenumber space gives the current magnitude and direction. In this case Eq. (5) can be solved using the least square method, over points $k_{j}$ with coherence (2) exceeding some pre-assigned threshold value (Coh $=0.8$ in present calculations).

For unknown $h$, the least square method is unstable. Over areas with detected waves much longer than the water depth $(k h<<1)$, the problem can reduce to zero current (Eq. (4)) to provide an estimated bathymetry. The longer the waves, the more accurate is this procedure. The analysis now solely concentrates on the peak wavenumber $k_{p}$. The time-lagged data is not anymore necessary, as the wave frequency conserves:

$$
\begin{gathered}
\sqrt{g k_{p} \tanh \left(k_{p} h\right)}=\text { const }=\sqrt{g k_{0}}, \\
h\left(k_{p}\right)=\frac{1}{k_{p}} \operatorname{atanh} \frac{k_{0}}{k_{p}}
\end{gathered}
$$

where $k_{0}$ is the wave peak wavenumber in the deep water region.

To note,a surface current should not significantly impact the estimated depth. For a $1 \mathrm{~m} / \mathrm{s}$ flow, the term $\mathbf{k U}$ is generally much smaller than $k \sqrt{g h}$, down to 5-10 m water depth. 


\subsection{Algorithm Implementation}

Figure (2) provides the step-by-step algorithm to estimate the projection of the current velocity in the wave propagation direction. To illustrate the methodology, a Sentinel-2 image is considered (Fig. 2, b), acquired August, 27th, 2016 at 06:46 in the region of the Somali current. This current is flowing from the South, rotating clockwise near the Somali peninsula. This large scale current is generally well captured by altimeter measurements (GlobCurrent surface current data, www.globcurrent.org), as reported in Fig. 2, a. The current front is further clearly distinguished using MSI optical data, as colored waters from the Africa coast are very well delineated. A fragment of the frontal area is shown in Fig. 2, c, with two squared areas of size $1 \mathrm{~km} \times 1 \mathrm{~km}$ marked inside (2) and outside (1) the current. Zooming in (Fig. 2, d, h), the detected wavy patterns for these two areas are apparently quite different: a dominant wave system in the upper area, and a more entangled multi-modal wave structure inside the current. The respective coherence fields, Eq. (2), and phase shift spectra, Eq. (3), are shown in subplots (e)-(f) and (i)-(j). Signs of phase spectra indicate the propagation direction of detected wave systems. For clarity, only points with coherence level larger than 0.8 are considered. In our case $(d t>0)$, negative phase shift corresponds to the direction waves are propagating to.

The curves $U_{j}(k)$, using (6) along transects shown on Fig. 2, f, j (dashed lines), are plotted in Fig. 2, 1. A linear approximation, $U_{j}=$ const, is obtained. A negligible current is found around point 1, and a surface current estimate of about $1.7 \mathrm{~m} / \mathrm{s}$ is found for point 2 .

Planes $\left(F-F_{0}\right)$ for these two image fragments are presented in Fig. 2, g, 


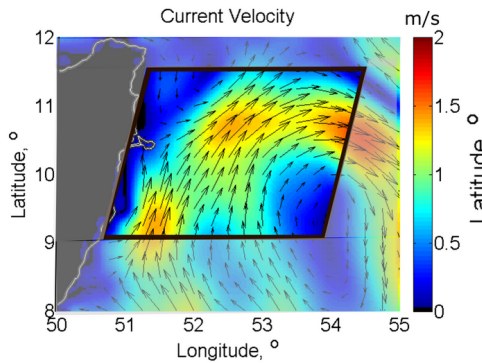

(a)

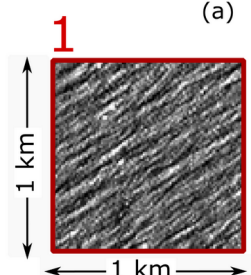

(d)

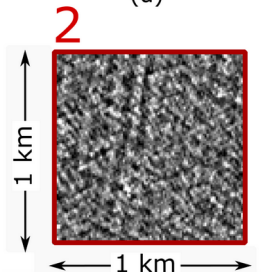

(h)

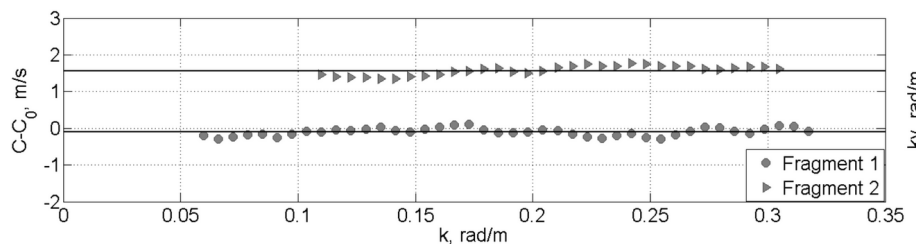

(I)

(b)

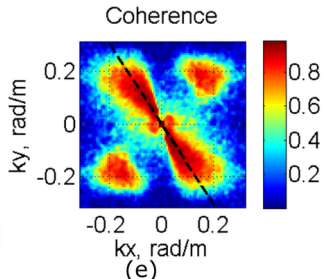

Phase shift
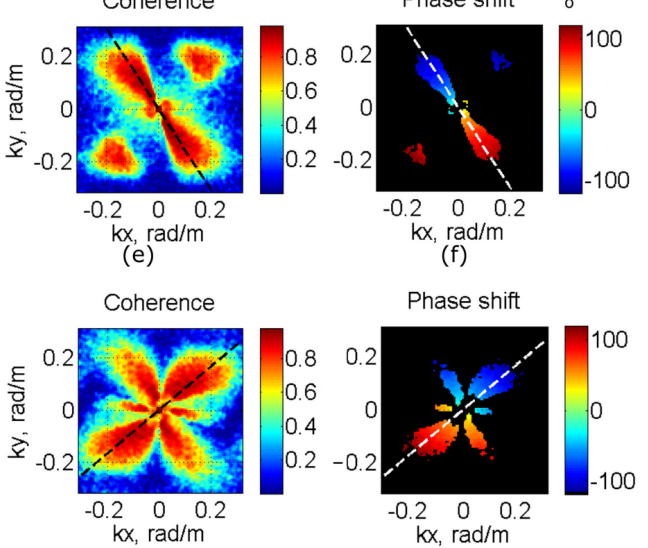

(i)

(j)
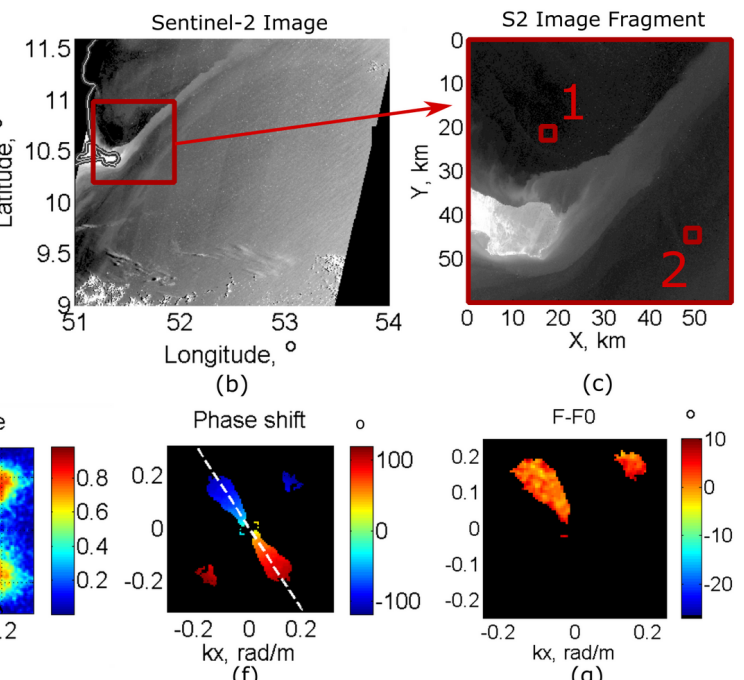

(g)

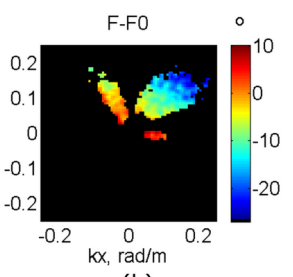

(k)

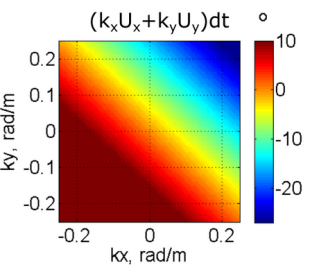

(m)

Figure 2: (a) Current field on 2016-08-27 near Somalia according to altimeter data; (b) MSI Sentinel-2 image, obtained on 2016-08-27, 06:46; (c) image fragment with current front manifestation; (d) zoomed image fragment around point 1; (e) coherence for bands B02 and B04; (f) phase shift spectrum; (g) observed and theoretical phase difference; (hk) the same as (d)-(g) but for fragment 2; (l) current velocity retrieval for the directions marked with dashed lines in (f) and $(\mathrm{j}) ;(\mathrm{m})$ plane approximation of $(F-F 0)$ plotted in $(\mathrm{k})$. 
k. The distribution of $F-F_{0}$ is almost uniform and close to zero for the point outside the current.It is significantly inclined in the second case. Using the least square method (Fig. 2, m) to retrieve the phase plane, the corresponding values of current components are $U_{x}=1.26 \mathrm{~m} / \mathrm{s}$ and $U_{y}=1.19 \mathrm{~m} / \mathrm{s}$.

\section{Results}

\subsection{Ocean Surface Current}

Repeated over other image fragments, where waves are clearly distinguished, this procedure helps to reconstruct a current field throughout the satellite observation areas. As obtained, the resulting current field, shown in Fig. 3, a, is in a good qualitative agreement with altimeter derived estimates, Fig. 2, a, and the detected large scale brightness features tracing the current front location, Fig. 2, b. Quantitative comparisons are presented in Fig. (3), b, c. Respective correlation coefficients, given in figure titles, show high statistical significance of the result. Both retrieved current amplitude and direction variability generally follow altimeter-derived estimates. The retrieved flow is stronger than the time- and spatial-averaged altimeter derived one. This "overestimation" is also systematic for the other cases considered below. Besides some isolated false points, an artificial strip-like structure is apparent in Fig. 3, a. This is an indication of a small systematical error inside images taken from different clusters. This error can arise from both inter-band geolocation inaccuracy and the time lag estimate. As further analyzed in Section 4, the former factor is the most significant for Sentinel-2 images.

Examples of surface current reconstruction over other ocean regions, the 


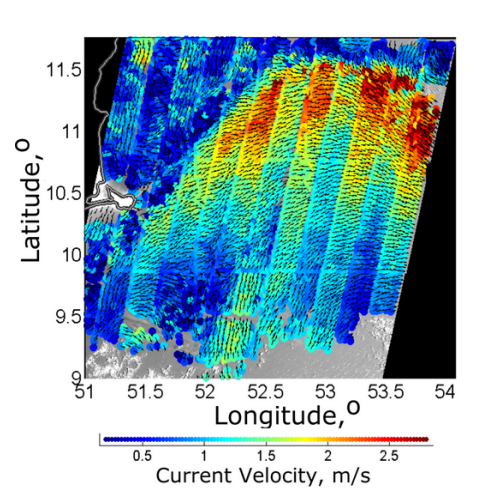

(a)

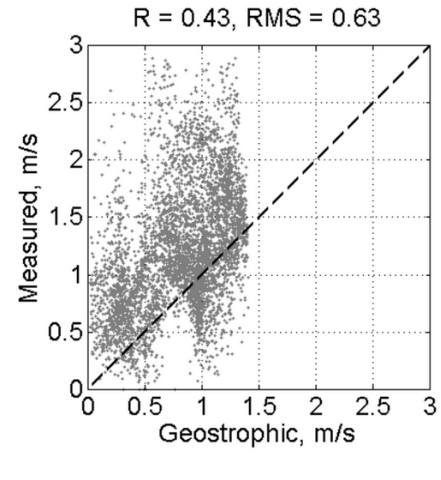

(b)

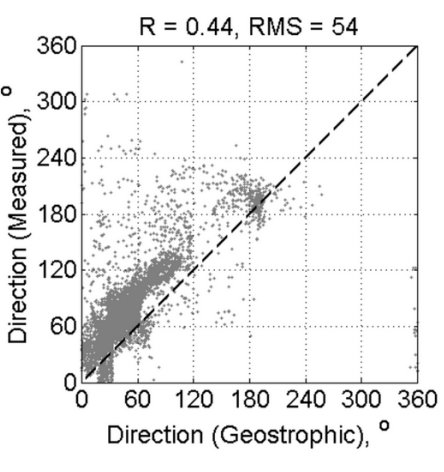

(c)

Figure 3: Current field retrieved from Sentinel-2 bands B02 and B04 for the case from Fig. 2 (a) and scatter plots of measured versus geostrophic current velocity (b) and direction (c).

Great Agulhas current system and the Gulf of Mexico, are further reported on Fig. 4. Again, at large scales, all retrieved current fields (Fig. 4, b) agree well with altimeter-derived geostrophic currents, (Fig. 4, a). At small spatial scales, the Sentinel-2 observations evidently capture more detailed structure and larger magnitudes in the main current core. This methodology essentially builds on spectral analysis of image brightness, and thus contemporaneously provides surface wave spectral information, i.e. peak wavelength and direction at every point. Retrieved wave fields are presented in Fig. 4, right. Although all analyzed cases correspond to multi-modal wave situations, an expected decrease/increase of the wavelength (with a corresponding increase/decrease of the wave amplitudes) of the same detected system, traveling opposite/along the current, is observed, i.e. comparison Fig. 4, bc. This is indicating strong wave-current interactions (Ardhuin et al., 2017; Quilfen et al., 2018).

As Fig. 3, scatter plots of measured vs altimeter-derived geostrophic cur- 
rent characteristics are presented in Fig. 4, d, e. Again, for the current magnitude, correlation coefficients are high $(>0.7)$, while retrieved currents are biased towards higher values, especially in the current core. Compare solid and dashed lines in Fig. 3, d, left, where all points and only points with $U<2 \mathrm{~m} / \mathrm{s}$ are taken, respectively. For the current direction, Fig. 3, e, correlation coefficients are less significant due to small angle variability. Still, estimates aggregate around the mean line, indicating the likely correctness of reconstructed directions.

\subsection{Bathymetry}

Another Sentinel-2 image, overlapping an intense current region, is reported on Fig. 5. This snapshot contains a part of the Gulf Stream, and was acquired on 2016-05-14, 15:59, over the Florida shelf Atlantic region. The part of the current field covered by the MSI image is consistent with altimeter-derived estimates, Fig. 5, a, d, e. The current frontal location is well-delineated and matches both altimeter gradients and brightness peculiarities on optical image (not shown). In line with previous results, the retrieved current is slightly stronger than the altimeter-derived one. Analysis also reveals an uniform flow of about $0.5 \mathrm{~m} / \mathrm{s}$, detected towards East-Southeast, probably associated to the tidal current, and thus not present in altimeter products.

In the shelf area, the total dispersion relation, accounting for the water depth, must be considered, Eq. (5), to estimate the flow velocity. Indeed, as obtained from Fig. 5, b, the waves traveling from the East are significantly modified by the bottom topography, shortening from $120 \mathrm{~m}$ in the East to 80 $\mathrm{m}$ at the coast. This measurable effect can help to solve the inverse problem, 
i.e. to estimate the ocean depth through Eq. (7). As anticipated, Fig. 5, c, f, display very good agreement of the reconstructed bathymetry and ETOPO1 Global Relief Model (www.ngdc.noaa.gov/mgg/global/).

\subsection{Small scale surface current}

All above examples confirm an overall qualitative agreement with largescale altimeter-derived estimates. As understood, these medium-resolution products cannot be used to evaluate detected fine structures of the retrieved current fields. A case, reported in Fig. 6, typically encompass large spatial variability of the surface current at small scales. Sentinel-2 MSI image was acquired on April, the 3rd 11:33, in North-East Atlantic, a region with intense tidal currents. MARC model (Modeling and Analysis for Coastal Research, http://marc.ifremer.fr/en/results/currents/), Fig. 6, a, forecasts currents up to $3 \mathrm{~m} / \mathrm{s}$, over the North-North-East part, around Ouessant Island, at the time of the acquisition. Strong current variability at small scales, O(1000 $\mathrm{m}$ ), is seemingly recovered with the proposed method, Fig. 6, b. Again, when results in neighbor strips systematically differ, it is likely indicating inter-channel geolocation imperfection in one or both clusters.

For more in-depth analysis, the accuracy of current reconstruction can be indirectly controlled by checking the expected frequency conservation law, following from kinematic equations for wave rays in the geometrical optics approximation (e.g. Phillips (1977)):

$$
\omega=\omega_{0}+\mathbf{k} \mathbf{U}=\text { const }
$$

where $\omega_{0}=\sqrt{g k \tanh (k h)}$ is the wave intrinsic frequency of surface water waves and $\omega$ is the detected frequency of the surface gravity wave propagating 
in a current region.

As obtained and reported on Fig. 6, c, $\omega_{0}$, estimated from the brightness peak wavenumber, strongly fluctuates in the region of intense non-uniform current. On the opposite, the total frequency $\omega_{0}+\mathbf{k} \mathbf{U}$, Fig. 6, d, is much smoother, in line with the current field reconstruction.

Differentiation of Eq. (8) reduces it to one-dimensional expressions, eliminating the unknown constant:

$$
\begin{aligned}
& -d \omega_{0} / d x=d\left(k_{x} U_{x}+k_{y} U_{y}\right) / d x \\
& -d \omega_{0} / d y=d\left(k_{x} U_{x}+k_{y} U_{y}\right) / d y
\end{aligned}
$$

In terms of wave-ray trajectories, an interesting consequence of these kinematic equations is the expected relation between the radius of curvature $R$ of the wave-rays, the wave group velocity $c_{g}$ and the vertical component of the current vorticity, assuming $c_{g}>>U$ (Kenyon, 1971):

$$
c_{g} / R=(\operatorname{rot} \mathbf{U})_{z}
$$

Both Eq. (9) and Eq. (10) can then be used to check the validity of all retrieved current fields. $U$ and $\omega_{0}$ derivatives can be estimated, using a least square method inside areas encompassing sufficient number of points, to ensure reliable surface current estimates. Ray curvature is estimated following the same procedure, through the derivatives of the wave vector direction $\alpha$ :

$$
\frac{1}{R}=\frac{\partial \alpha}{\partial r}=\frac{\partial \alpha}{\partial x} \cos \alpha+\frac{\partial \alpha}{\partial x} \sin \alpha
$$

The comparison between left and right parts of Eq. (9) and Eq. (10), for the cases shown in Fig. 3 and Fig. 4, is presented Fig. 7. Mean values of 
each parameter (gray dots) are calculated around every point inside small $0.2^{\circ}$ square image fragments. Results first appear highly scattered. This is mainly induced from unstable estimations of the derivatives and their combinations from spatially non-uniform fields. This is especially evident for cases with multiple wave systems for which the chosen wavenumber peak may belong to any of co-existing wave trains. The ray curvature is also very sensitive and difficult to evaluate (Eq. (11), Fig. 7, bottom) given the small dynamic range of wave direction values inside each squared area. Yet, binaveraging (magenta/purple squares) improves estimates, close to the mean expected one-to-one line (black). Overall, the retrieved current fields are thus generally consistent with the conservation law principle.

\subsection{Whitecap Velocities}

To further take advantage of the time-lagged Sentinel-2 MSI acquisitions, the direct displacements of moving coherent targets can also be performed. For ocean applications, a 1-s time-lag is sufficient to study motion and periodicity of whitecaps forming during wave breaking events (Donelan et al., 1972). In the example shown Fig. 8, upper line, fragments from different parts of the Sentinel-2 observations, considered in Fig. 2 and Fig. 3 (Somali), are combined to construct RGB-images. The red color corresponds to the initial signal, i.e. adjusted B02 or B04 brightness. The green color corresponds to the same signal, acquired $1 \mathrm{~s}$ latter. The blue color corresponds to the averaged signal. Whitecaps are usually smaller than Sentinel-2 pixel resolution $(10 \mathrm{~m})$, but wave breaking events are clearly detected. Motions of wave breaking fronts can then be obtained. Inter-channel correlation function provides object displacement and thus, their velocity and direction (Kubryakov 
et al. (2018)). Peak positions of the resulting correlation functions, shown in Fig. 8, f-j, correspond to the mean direction of the whitecap propagation. The estimated velocities range between $5 \mathrm{~m} / \mathrm{s}$ and $8 \mathrm{~m} / \mathrm{s}$, comparable to the phase and group velocities of the dominant waves, about $9 \mathrm{~m} / \mathrm{s}$ and $4.5 \mathrm{~m} / \mathrm{s}$, respectively.

\section{Discussion: Method Applicability and Accuracy}

This methodology to infer surface current velocity shall apply to any space-borne optical multi-channel images, having inter-channel time-lag of order of seconds, and sufficient spatial resolution to resolve surface waves. A necessary condition is indeed the visibility of surface wave modulations to analyse their propagation properties. With pixel resolution of order 10 $\mathrm{m}$, this is usually fulfilled in most of the sun glint area. Except within the very specular part of the glint or too far from it, surface waves are well detected. Other factors can prevent the analysis of wave dispersion characteristics: clouds, surface roughness features like slicks, ship tracks or objects on the sea surface, including bright whitecaps. Whitecaps are often present in frontal current regions, and not solely during windy conditions. Yet, the methodology appears quite robust. As demonstrated, Fig. 3 and Fig. 7, a (Somali case), a surface current field was retrieved under high wind conditions with numerous detectable whitecaps. The retrieved current values may then be overestimated over the most active wave breaking area (NorthEast part). Future investigations shall thus be necessary to better assess possible biases induced by visible whitecaps.

Results also depend on the directional spread of the wave spectrum, and 
the relative wave-current direction. Waves propagating perpendicular to a current direction are only weakly impacted. The procedure described in Section 2 can not apply. Ideally, wave systems exhibiting a large directional spread will help. Technically, a solution is to design an instrument with increased multi-angle bi-static detector capability, to combine high angular sampling with high spatial resolution (Rascle et al. (2018)).

Accurate estimation directly depends on the time-lag between acquisitions, and their co-registration error. To retrieve surface wave propagation characteristics, time-lags should be long enough to provide a reliable crossspectral phase estimate, but short enough to ensure strong coherency between the two consecutive observations. For a time shift $d t$ and displacement $d x$, with systematic errors $\Delta t$ and $\Delta x$, respectively, the velocity estimate error is

$$
\Delta v=v-\frac{v d t+\Delta x}{d t+\Delta t}=v \frac{\Delta t}{d t+\Delta t}-\frac{\Delta x}{d t+\Delta t}
$$

The first term in (12) r.h.s. indicates the time-lag uncertainty. The second one is the image co-registration error. Time-lag uncertainty increases with decreasing $d t$, i.e. the larger the time-lag the better the accuracy of the velocity estimation. Though not equivalent, spatial and temporal errors can occasionally compensate each other: a systematic displacement will appear as an additional current. A constant $\Delta x / d t$ adds to the estimated phase velocity $c(k)$, and thus to the respective current projection. A temporal error distorts $c(k)$ with a coefficient $d t /(d t+\Delta t)$. The influence of both terms in (12) is shown in Fig. 9, for $1 \mathrm{~s}$ and $2 \mathrm{~s}$ inter-band time-lags. For the bands with 10 $\mathrm{m}$ resolution and $1 \mathrm{~s}$ time-lag, a co-registration accuracy better than $0.1 \mathrm{~m}$ 
(0.01 pixel) is required to ensure reliable velocity estimate, down to $0.1 \mathrm{~m} / \mathrm{s}$. Examples in Section 3 demonstrate that surface currents down to $0.3 \mathrm{~m} / \mathrm{s}$ are correctly retrieved, except for the case shown on Fig. 6. For this case, the current magnitudes from neighbor strips systematically differ by about $1 \mathrm{~m} / \mathrm{s}$. Accordingly, B02-B04 inter-band matching accuracy is better than 0.1 pixel, and for most cases, better than 0.03 pixel. MSI co-registration accuracy seems to outclass the values listed in the Sentinel-2 data quality report, where 0.168 pixel is stated for B02 and B04 (sentinel.esa.int).

Increasing the time-lag reduces the error (green line). But, a 5\% time-lag error leads to $0.5 \mathrm{~m} / \mathrm{s}$ uncertainty in velocity estimate for $50 \mathrm{~m}$ waves. In our present analysis, $d t$ has been estimated with an accuracy of about $1 \%$ (Section 2). The associated error is then negligible compared to the error related with co-registration precision.

\section{Conclusion}

Satellite sun glint images of the sea surface often provide highly powerful means to observe and quantify small-scale spatial variations of currents, wind and surfactants. In this paper, the Sentinel-2 MultiSpectral Instrument properties and capabilities are further demonstrated.. Sentinel-2 instruments collect multiple spectral band images, corresponding to specific sensing wavelength and spatial resolution, i.e. $10 \mathrm{~m}, 20 \mathrm{~m}$, and $60 \mathrm{~m}$. Because images are acquired one at the time, with a fixed time-lag between observations, spatiotemporal characteristics of propagating ocean surface waves can uniquely be retrieved, including motions of whitecaps.

A method is applied to estimate the surface current from images of the sea 
surface, taken from two particular Sentinel-2 bands with a time delay of order 1 second. The measured deviation from the expected linear surface wave dispersion relationship directly relates to surface current impacts. The waverays, wavevenumber and dispersion fields, $w(\mathbf{k})$, can cover a fairly wide range of angles to serve the reconstruction of the total surface current vector. In the absence of current, and shallow water conditions, the dispersion variations are determined by the ocean depth variations.

For MSI, the largest time-lag between acquisitions using 10-m resolution bands, is of order $1 \mathrm{~s}$. To improve surface current determination, optimized acquisitions could consider video-like acquisitions, rather than two snapshots. Already, the reconstructed current fields appear valuable. In absence of ground-truth information, results well compare, at large to medium spatial scales, with altimeter-derived geostrophic current estimates. At higher resolution, the analysis satisfy conservation laws, as obtained from wave-ray kinematic equations.

The possibility to estimate the speed and direction of large whitecaps, has also been tested. Again, this is opening for future investigations, as a potential key capability to infer direct measurements of momentum and gas fluxes.

As discussed for reliable current reconstruction, MSI images should be co-registered with an accuracy better than 0.03 pixel. For Sentinel-2 MSI measurements, this is generally fulfilled.

Overall, these different results demonstrate very encouraging prospects to combine different satellite measurements (altimeter, optical, radar). The design of dedicated future satellite missions to precisely retrieve surface cur- 
rents at high resolution, may thus build upon a satellite constellation, building from standard altimeter data, to medium resolution measurements (future SWOT mapping altimeter), completed with multi-angle bi-static optical measurements (Kudryavtsev et al. (2012); Rascle et al. (2018)). Combining high angular diversity and pixel spacing, multi-angle optical measurements could also include polarization capabilities to help separate sunglint radiance from water-leaving, skyglint, and whitecap contributions (Harmel and Chami (2013)). While limited to favorable day-light and cloud-less conditions, Sentinel-2 MSI observations already offer essential means for multivalidation purposes: local wind, waves and current transformations and interactions. Future investigations are necessary to better assess biases, possibly induced by detected whitecaps. To note, the combined capability to track whitecaps and surface wave field transformation shall also further be explored to map and inform complex wave-current interactions (Rascle et al. (2014); Ardhuin et al. (2017)) in the vicinity of ocean fronts and coastal areas.

\section{Acknowledgments}

The authors are grateful to Vladimir Dulov for the discussion and useful comments, and to anonymous Reviewers whose valuable suggestions helped to improve the paper. The work was supported by Russian Science Foundation via grant 17-77-30019 and the Ministry of Education and Science of the Russian Federation under the State Assignment No. 0827-2018-0003, also supported through the ESA projects SArONG under contract 4000117644/16/NL/FF/gp and GlobCurrent under contract 4000109513/13/ILG. The Copernicus Sen- 
tinel2 data used in this paper are available at: https://scihub.copernicus.eu/dhus/\#/home

\section{References}

Ardhuin, F., Gille, S. T., Menemenlis, D., Rocha, C. B., Rascle, N., Chapron, B., Gula, J., and Molemaker, J. (2017). Small-scale open ocean currents have large effects on wind wave heights. Journal of Geophysical Research: Oceans, 122(6):4500-4517.

Barber, N. F. (1949). A Diffraction Analysis of a Photograph of the Sea. Nature, 164(485).

Chapron, B., Collard, F., and Ardhuin, F. (2005). Direct measurements of ocean surface velocity from space: Interpretation and validation. Journal of Geophysical Research: Oceans, 110(C7).

De Michele, M., Leprince, S., Thiebot, J., Raucoules, D., and Binet, R. (2012). Direct measurement of ocean waves velocity field from a single spot-5 dataset. Remote Sensing of Environment, 199:266-271.

Donelan, M., Longuet-Higgins, M. S., and Turner, J. S. (1972). Periodicity in whitecaps. Nature, 239(5373):449-451.

Dugan, J. and Piotrowski, C. (2003). Surface current measurements using airborne visible image time series. Remote Sensing of Environment, 84:309-319.

Dugan, J. P., Piotrowski, C. C., and Williams, J. Z. (2001). Water depth and surface current retrievals from airborne optical measurements of sur- 
face gravity wave dispersion. Journal of Geophysical Research: Oceans, 106(C8):16903-16915.

Fedele, F., Benetazzo, A., Gallego, G., Shih, P.-C., Yezzi, A., Barbariol, F., and Ardhuin, F. (2013). Space-time measurements of oceanic sea states. Ocean Modelling, 70:103-115.

Goldstein, R. and Zebker, H. (1987). Interferometric radar measurement of ocean surface currents. Nature, pages 707-709.

Hansen, M., Kudryavtsev, V., Chapron, B., Johannessen, J., Collard, F., Dagestad, K.-F., and Mouche, A. (2012). Simulation of radar backscatter and doppler shifts of wave-current interaction in the presence of strong tidal current. Remote Sensing of Environment, 120:113 - 122. The Sentinel Missions - New Opportunities for Science.

Harmel, T. and Chami, M. (2013). Estimation of the sunglint radiance field from optical satellite imagery over open ocean: Multidirectional approach and polarization aspects. Journal of Geophysical Research: Oceans, 118(1):76-90.

Johannessen, J. A., Chapron, B., Collard, F., Kudryavtsev, V., Mouche, A., Akimov, D., and Dagestad, K.-F. (2008). Direct ocean surface velocity measurements from space: Improved quantitative interpretation of envisat asar observations. Geophysical Research Letters, 35(22).

Kääb, A., Lamare, M., and Abrams, M. (2013). River ice flux and water velocities along a $600 \mathrm{~km}$-long reach of lena river, siberia, from satellite stereo. Hydrology and Earth System Sciences, 17(11):4671-4683. 
Kääb, A. and Leprince, S. (2014). Motion detection using near-simultaneous satellite acquisitions. Remote Sensing of Environment, 154:164-179.

Kenyon, K. E. (1971). Wave refraction in ocean currents. Deep Sea Research and Oceanographic Abstracts, 18(10):1023 - 1034.

Kubryakov, A., Plotnikov, E., and Stanichny, S. (2018). Reconstructing large- and mesoscale dynamics in the black sea region from satellite imagery and altimetry data. a comparison of two methods. Remote Sensing, $10(2)$.

Kudryavtsev, V., Myasoedov, A., Chapron, B., Johannessen, J. A., and Collard, F. (2012). Imaging mesoscale upper ocean dynamics using synthetic aperture radar and optical data. Journal of Geophysical Research: Oceans, 117(C4):n/a-n/a. C04029.

Kudryavtsev, V., Yurovskaya, M., Chapron, B., Collard, F., and Donlon, C. (2017a). Sun glitter imagery of ocean surface waves. Part 1: Directional spectrum retrieval and validation. Journal of Geophysical Research: Oceans, 122(2):1369-1383.

Kudryavtsev, V., Yurovskaya, M., Chapron, B., Collard, F., and Donlon, C. (2017b). Sun glitter imagery of surface waves. Part 2: Waves transformation on ocean currents. Journal of Geophysical Research: Oceans, 122(2):1384-1399.

Leckler, F., Ardhuin, F., Peureux, C., Benetazzo, A., Bergamasco, F., and Dulov, V. (2015). Analysis and Interpretation of Frequency-Wavenumber 
Spectra of Young Wind Waves. Journal of Physical Oceanography, $45(10): 2484-2496$.

Martin, A. C. and Gommenginger, C. (2017). Towards wide-swath highresolution mapping of total ocean surface current vectors from space: Airborne proof-of-concept and validation. Remote Sensing of Environment, $197: 58-71$.

Martin, A. C., Gommenginger, C. P., and Quilfen, Y. (2018). Simultaneous ocean surface current and wind vectors retrieval with squinted sar interferometry: Geophysical inversion and performance assessment. Remote Sensing of Environment, 216:798 - 808.

Matthews, J. and Awaji, T. (2010). Synoptic mapping of internal-wave motions and surface currents near the lombok strait using the along-track stereo sun glitter technique. Remote Sensing of Environment, 114(8):1765 $-1776$.

Matthews, J. P. and Yoshikawa, Y. (2012). Synergistic surface current mapping by spaceborne stereo imaging and coastal hf radar. Geophysical Research Letters, 39(17).

Phillips, O. M. (1977). The Dynamics of the Upper Ocean. Cambridge University Press, New York, 2 edition.

Quilfen, Y., Yurovskaya, M., Chapron, B., and Ardhuin, F. (2018). Storm waves focusing and steepening in the agulhas current: Satellite observations and modeling. Remote Sensing of Environment, 216:561 - 571. 
Rascle, N., Chapron, B., Ponte, A., Ardhuin, F., and Klein, P. (2014). Surface roughness imaging of currents shows divergence and strain in the wind direction. Journal of Physical Oceanography, 44(8):2153-2163.

Rascle, N., Molemaker, J., Marie, L., Nouguier, F., Chapron, B., Lund, B., and Mouche, A. (2017). Intense deformation field at oceanic front inferred from directional sea surface roughness observations. Geophysical Research Letters, 44(11):5599-5608. 2017GL073473.

Rascle, N., Nouguier, F., Chapron, B., and Ocampo-Torres, F. J. (2018). Sunglint images of current gradients at high resolution: Critical angle and directional observing strategy. Remote Sensing of Environment, 216:786 797.

Rebecq, H., Gallego, G., Mueggler, E., and Scaramella Perez, D. (2017). Emvs: Event-based multi-view stereo - 3d reconstruction with an event camera in real-time. International Journal of Computer Vision, pages 121.

Rodriguez, E., Wineteer, A., Perkovic-Martin, D., Gál, T., Stiles, B., Niamsuwan, N., and Rodriguez Monje, R. (2018). Estimating ocean vector winds and currents using a Ka-band pencil-beam doppler scatterometer. 10. 576 .

Romeiser, R., Runge, H., Suchandt, S., Kahle, R., Rossi, C., and Bell, P. S. (2014). Quality assessment of surface current fields from terrasar-x and tandem-x along-track interferometry and doppler centroid analysis. IEEE Transactions on Geoscience and Remote Sensing, 52(5):2759-2772. 
Takasaki, K., Sugimura, T., and Tanaka, S. (1994). Speed vector measurement of moving objects using jers-1/ops data. Journal of The Remote Sensing Society of Japan, pages 213-219.

Yurovskaya, M., Kudryavtsev, V., S. Shirokov, A., and Yu. Nadolya, I. (2018a). Field measurements of the sea surface wave spectrum from photos of sunglitter taken from drone. Sovremennye problemy distantsionnogo zondirovaniya Zemli iz kosmosa, 15:245-257.

Yurovskaya, M., Kudryavtsev, V. N., Chapron, B., Rascle, N., and Collard, F. (2018b). Wave spectrum and surface current retrieval from airborne and satellite sunglitter imagery. In 2018 IEEE International Geoscience and Remote Sensing Symposium, IGARSS 2018, Valencia, Spain, July 22-27, 2018, pages 3192-3195.

Yurovskaya, M., Rascle, N., Kudryavtsev, V., Chapron, B., Marié, L., and Molemaker, J. (2018). Wave spectrum retrieval from airborne sunglitter images. Remote Sensing of Environment, 217:61-71.

Yurovsky, Y. Y., Kudryavtsev, V. N., Grodsky, S. A., and Chapron, B. (2019). Sea surface ka-band doppler measurements: Analysis and model development. Remote Sensing, 11(7). 

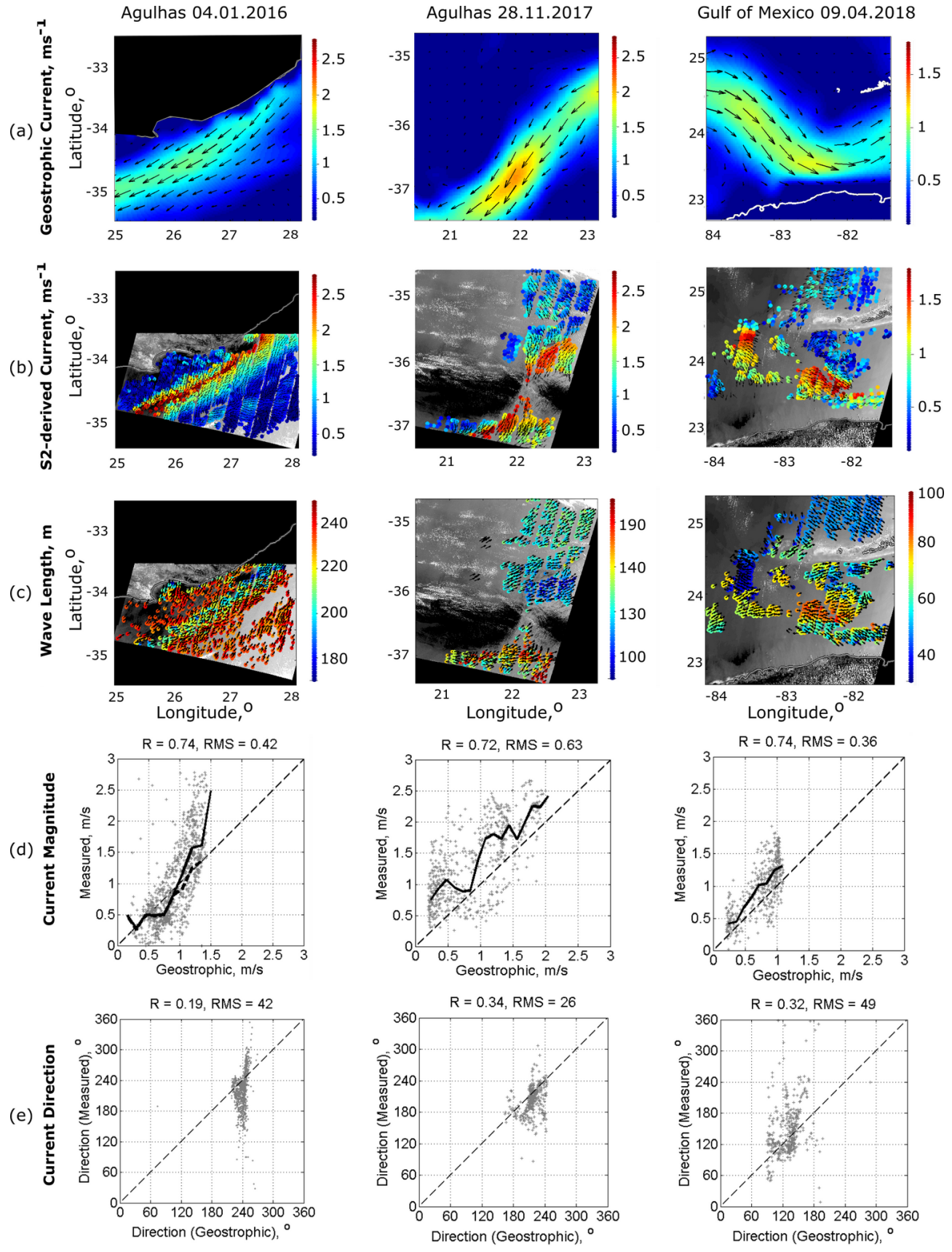

Figure 4: (a) Geostrophic current from altimeter data in the region of Agulhas current on the 4th of January, 2016 (left) and 28th of November, 2017 (center), and the Gulf of Mexico on 2018-04-09 (right); (b) respective surface currents retrieved from Sentinel-2 MSI images; (c) wavelengths and directionggeconstructed from brightness spectra peak wavenumber; (d)-(e) scatter plots of measured versus geostrophic current velocity and direction (black solid lines show interval-averaged values, dashed line in (d), left, is the same, but for points $U<2 \mathrm{~m} / \mathrm{s})$. 


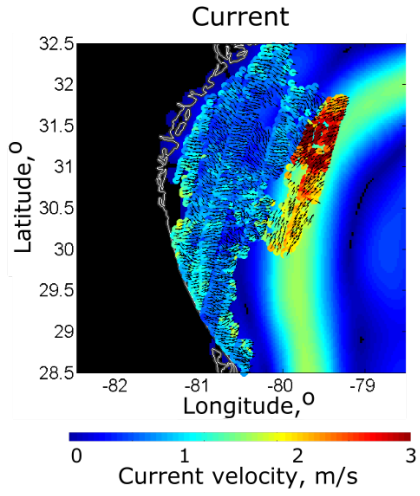

(a)

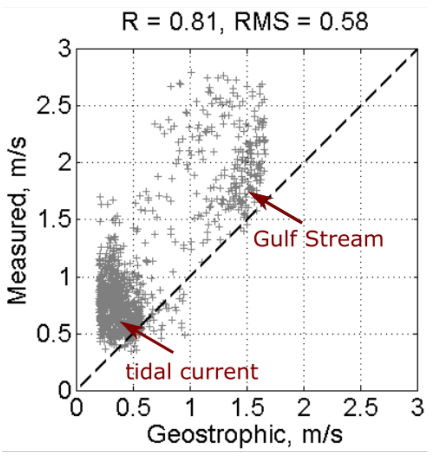

(d)

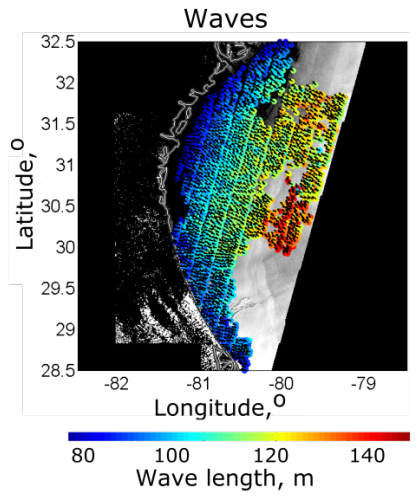

(b)

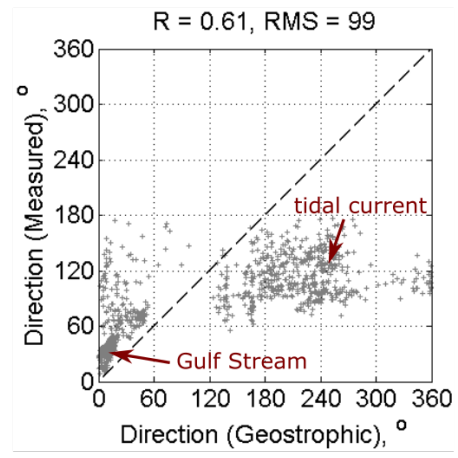

(e)

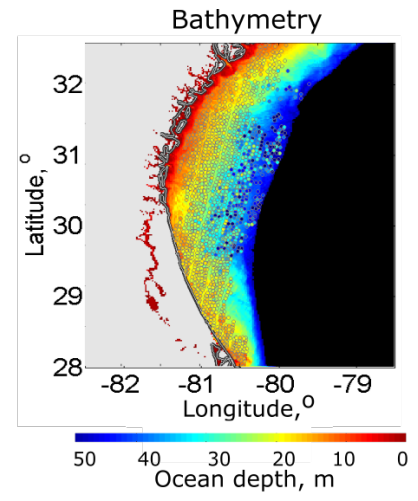

(c)

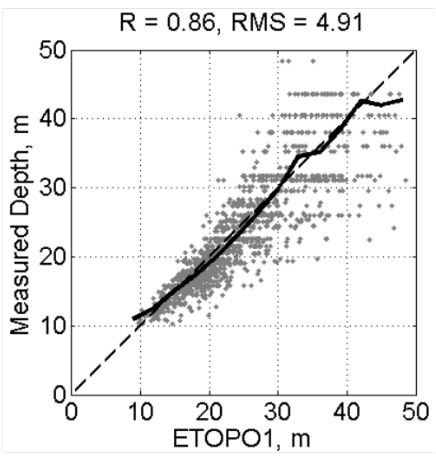

(f)

Figure 5: (a) MSI-derived current field (points with arrows) overlapping geostrophic current field in West Atlantic on the 14th of May, 2016; (b) wavelengths and directions obtained from Sentinel-2 image brightness; (c) ocean depth reconstructed through the wave dispersion relation from MSI image (dots) and ETOPO1 data (background); (d)-(e) scatter plots of geostrophic vs measured current velocity and direction; (f) scatter plot of ETOPO1 bathymetry vs reconstructed ocean depth. 

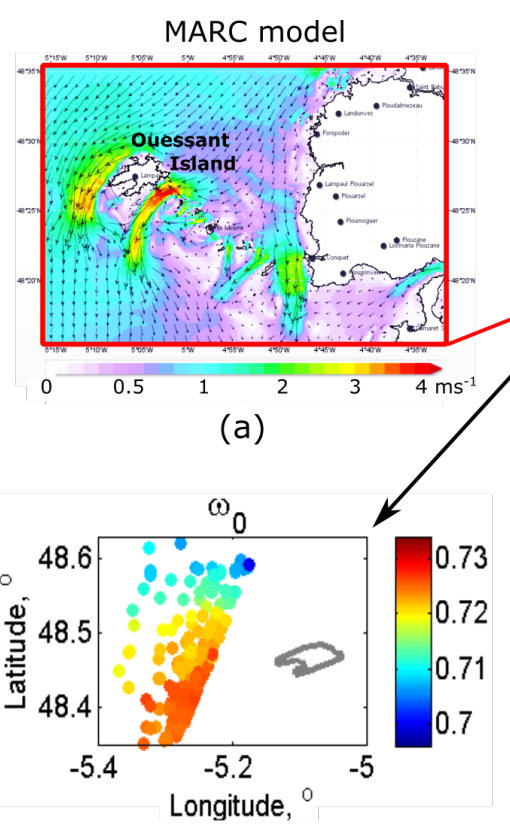

(c)

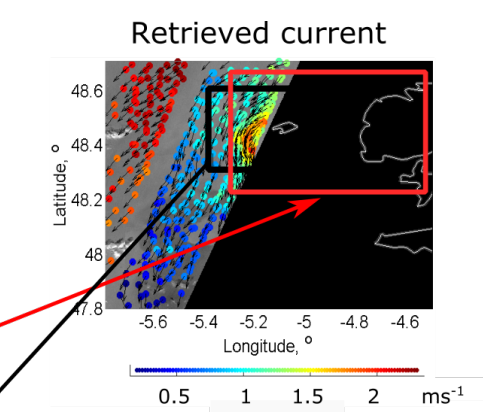

(b)

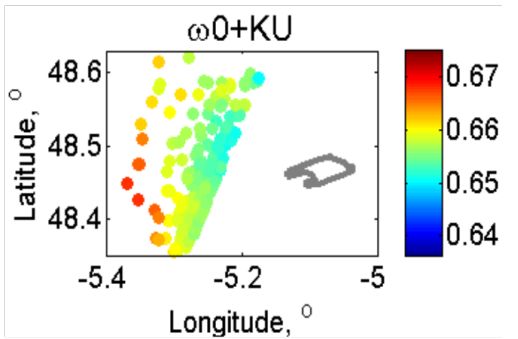

(d)

Figure 6: (a) Tidal current in the North of Biscay Bay on the 3rd of April, 11:30, according to MARC model; (b) current reconstructed from Sentinel-2 snapshot obtained on the 3rd of April, 11:33; (c) wave frequencies derived from image brightness spectra; (d) total wave frequency, $\omega_{0}+\mathrm{kU}$, calculated using MSI-derived current vectors. 


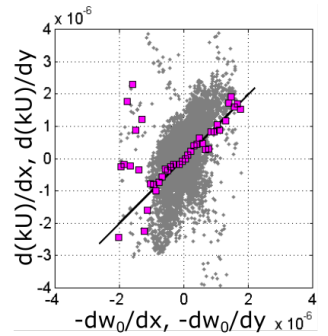

(a)

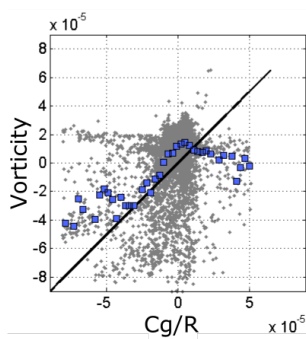

(e)

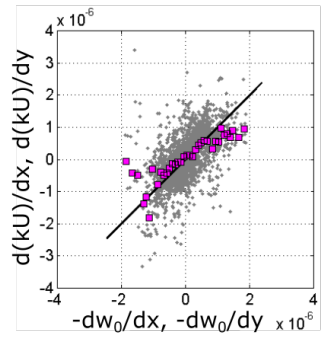

(b)

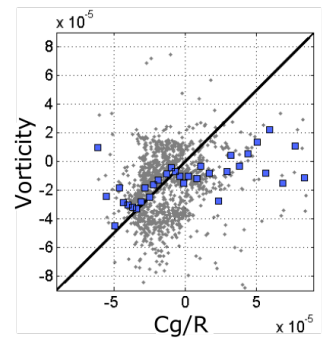

(f)

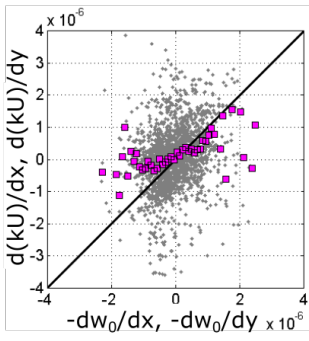

(c)

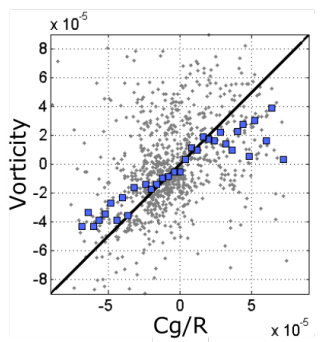

(g)

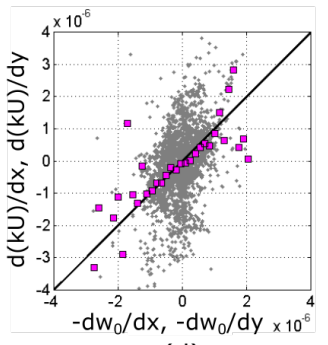

(d)

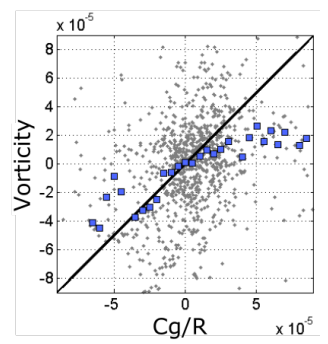

(k)

Figure 7: (a)-(d) left and right parts of Eq. 9 for every point (gray dots) in Fig. 3 and Fig. 4 (Somali current (a), Agulhas (b)-(c) and Gulf of Mexico (d)); squares are interval-averaged data; (e)-(k) left and right parts of Eq. 10 for the same cases as in (a)-(d). 


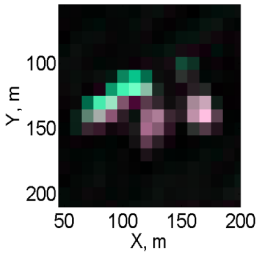

(a)

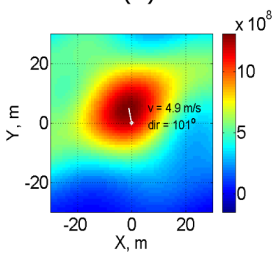

(f)

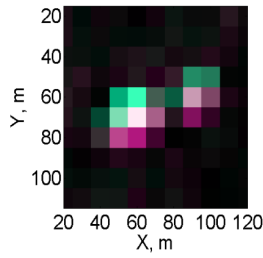

(b)

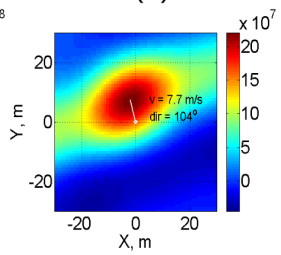

(g)

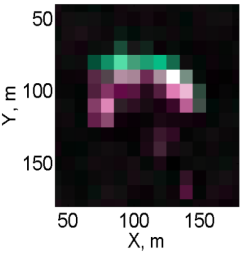

(c)

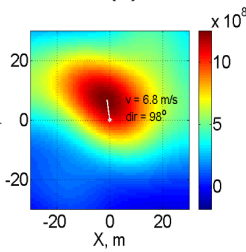

(h)

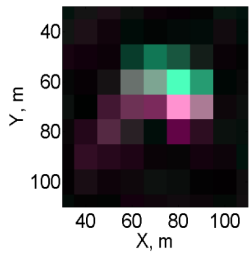

(d)

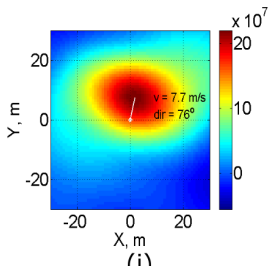

(i)

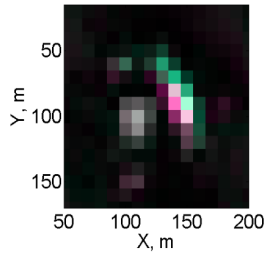

(e)

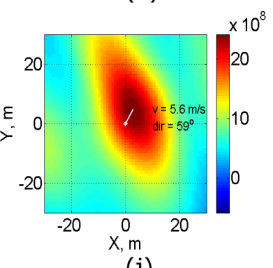

(j)

Figure 8: (a)-(e) RGB-compositions of MSI image fragments containing manifestation of wave breaking events. Red (earlier) and green (later) are the modified brightness signals of Sentinel-2 channels B02/B04, blue is their average, time lag is $1 \mathrm{~s}$; (f)-(j) corresponding correlation functions; the location of maximum indicates event speed and direction. 


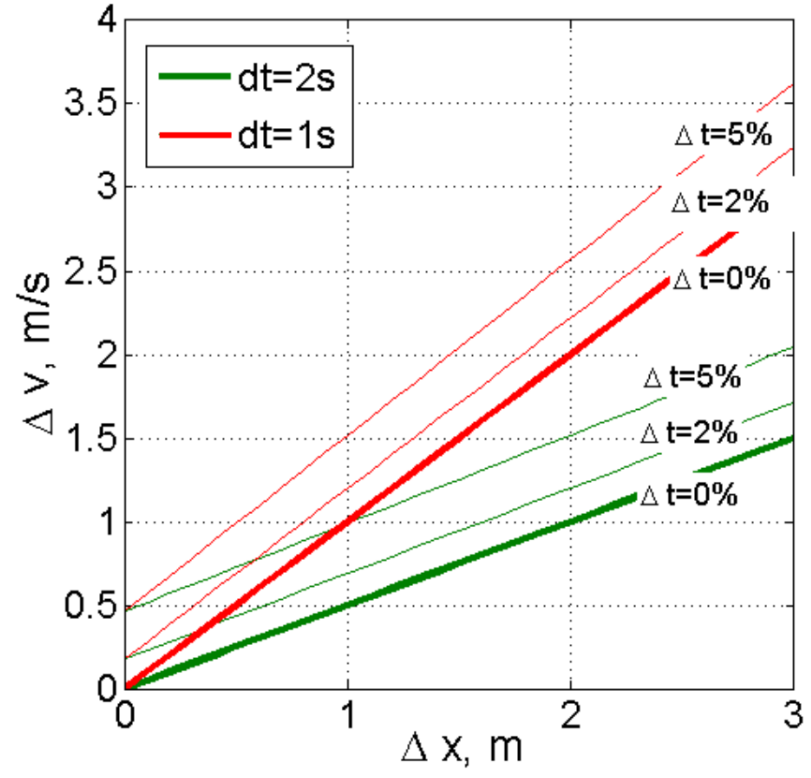

Figure 9: The absolute error of velocity estimate for $1 \mathrm{~s}$ and $2 \mathrm{~s}$ time lags (color) and different time lag estimation error $\Delta t$ (for $50 \mathrm{~m}$ waves), and inter-channel matching accuracy $\Delta x . \Delta x$ and $\Delta t$ are of different signs here. 